\title{
In vitro and in vivo therapeutic efficacy of CXCR4 antagonist BKT140 against human non-small cell lung cancer
}

\author{
Duha Fahham, MSc, ${ }^{\text {a } I d o ~ D . ~ W e i s s, ~ P h D, ~}{ }^{\text {a }}$ Michal Abraham, PhD,${ }^{\mathrm{a}, \mathrm{b}}$ Katia Beider, $\mathrm{PhD},{ }^{\mathrm{a}}$ Wald Hanna, ${ }^{\mathrm{a}}$ \\ Zippora Shlomai, MSc, ${ }^{\mathrm{c}}$ Orly Eizenberg, PhD, ${ }^{\mathrm{b}}$ Gideon Zamir, MD, ${ }^{\mathrm{c}}$ Uzi Izhar, MD, ${ }^{\mathrm{d}}$ Oz M. Shapira, MD, ${ }^{\mathrm{d}}$ \\ Amnon Peled, $\mathrm{PhD},{ }^{\mathrm{a}}$ and Ori Wald, $\mathrm{MD}, \mathrm{PhD}^{\mathrm{a}, \mathrm{d}}$
}

\begin{abstract}
Objectives: CXCR4/CXCL12 interactions promote non-small cell lung cancer (NSCLC) growth and dissemination. Furthermore, this axis might promote NSCLC resistance to chemotherapy and/or radiotherapy. Therefore, the CXCR4/CXCL12 axis constitutes an attractive therapeutic target for the treatment of NSCLC. We aimed to characterize the therapeutic efficacy of the novel CXCR4 antagonist BKT140 against human NSCLC.

Methods: We determined the CXCR4 expression in 5 NSCLC cell lines (H358, A549, H460, H1299, and L4). We then tested the colony-forming capacity and proliferation of these cells in the presence of CXCL12 and BKT140. Next, we measured the in vivo growth of A549 and H460 xenografts with or without BKT140 treatment. Finally, we examined, in vitro, the potential antiproliferative effect of BKT140 combined with cisplatin or paclitaxel and after irradiation of NSCLC cells.
\end{abstract}

Results: All tested cell lines expressed CXCR4 and showed increased colony formation in response to CXCL12 stimulation. BKT140 reduced the colony-forming capacity of NSCLC cells. Proliferation assays demonstrated both cytotoxic and cytostatic properties for this peptide. H460 cells were the most sensitive to BKT140 and A549 cells the least. Subcutaneous administration of BKT140 significantly delayed the development of H460 xenografts and showed a similar trend for A549 xenografts. Finally, the antiproliferative effects of BKT140 appears to be additive to those of chemotherapeutic drugs and radiotherapy.

Conclusions: Targeting the CXCL12/CXCR4 axis with BKT140 attenuated NSCLC cells tumor growth and augmented the effects of chemotherapy and radiotherapy. Future research will benefit from delineating the downstream mechanism of BKT140 action and defining BKT140 susceptibility markers. (J Thorac Cardiovasc Surg 2012;144:1167-75)

\section{Supplemental material is available online.}

Despite advances in surgery, chemotherapy, and radiotherapy during the past decades, the death rate from lung cancer remains a global health problem. ${ }^{1}$ It was estimated that more than 220,000 new cases of lung cancer would be diagnosed in the United States in 2010. Lung cancer-related mortality was anticipated to peak at more than 150,000 cases/y, and the reported overall 5-year survival rate remains at only

\footnotetext{
From the Goldyne Savad Institute of Gene Therapy, ${ }^{a}$ Laboratory for Surgical Research, ${ }^{\mathrm{c}}$ and Department of Cardiothoracic Surgery, ${ }^{\mathrm{d}}$ Hadassah University Hospital, Jerusalem, Israel; and Biokine Therapeutics, ${ }^{\mathrm{b}}$ Rehovot, Jerusalem, Israel.

This work was supported by the Israeli Science Foundation Morasha research grant program.

Disclosures: Authors have nothing to disclose with regard to commercial support.

Read at the 92nd Annual Meeting of The American Association for Thoracic Surgery, San Francisco, California, April 28-May 2, 2012.

Received for publication March 27, 2012; revisions received June 27, 2012; accepted for publication July 25, 2012; available ahead of print Aug 27, 2012.

Address for reprints: Ori Wald, MD, PhD, Department of Cardiothoracic Surgery, Hadassah University Hospital, PO Box 12000, Jerusalem, Israel (E-mail: ori.wald@ mail.huji.ac.il).

0022-5223/\$36.00

Crown Copyright (C) 2012 Published by Elsevier Inc. on behalf of The American Association for Thoracic Surgery

http://dx.doi.org/10.1016/j.jtcvs.2012.07.031
}

$15 \% .^{2}$ Thus, new treatment strategies for lung cancer patients are urgently needed. One attractive strategy to follow is targeting the chemokine receptor CXCR4. ${ }^{3}$

CXCR4 is a key driver of solid malignant propagation, in general, and of non-small cell lung cancer (NSCLC), in particular. $^{4,5}$ Two lines of evidence support this claim. The first comes from retrospective studies of human metastatic cancers, in which high CXCR4 expression levels were associated with a worse prognosis. The second comes from animal experimental models demonstrating an aggressive phenotype for high CXCR4-expressing tumor cells, suggesting that blockage of the CXCR4/CXCL12 axis inhibits metastasis and enhances survival. ${ }^{4,6-8}$ Clinical observational studies have demonstrated that high CXCR4 expression in NSCLC tumors was associated with greater tumor dimensions (higher $\mathrm{T}$ score) and an increased risk of disease recurrence (reduced disease-free interval). ${ }^{9-11}$ Furthermore, in vivo experimental models have demonstrated that blocking CXCR4 reduced NSCLC's metastatic potential. ${ }^{12}$ In addition, recent research has suggested that CXCR4/CXCL12 interactions in the NSCLC tumor microenvironment might act in autocrine and paracrine manners to enhance primary tumor growth and could also contribute to lung cancer immune evasion by inducing the recruitment/retention of CXCR4+ 


\section{Abbreviations and Acronyms \\ $\mathrm{FCS}=$ fetal calf serum \\ NSCLC $=$ non-small cell lung cancer \\ RPMI = Roswell Park Memorial Institute}

FoxP3+ cells into the tumor microenvironment. ${ }^{13-15}$ Additional studies have suggested that expression and activation of CXCR4 in lung cancer cells and stem cells is associated with an increase of resistance to both chemotherapeutic drugs and radiotherapy. ${ }^{16,17}$ Taken together, these data advocate that targeting CXCR4 might alter various key pathologic processes involved in NSCLC disease propagation.

In the present study, we focused our attention on BKT140, a novel, small peptide with CXCR4-antagonistic properties. ${ }^{18}$ BKT140 was initially developed as an antihuman immunodeficiency virus agent, chemically structured to block the entry of the CXCR4 tropic human immunodeficiency virus into CXCR4-expressing T cells. ${ }^{19,20}$ The affinity of BKT140 for CXCR4 was tested using iodine125 radiolabeled CXCL12, a specific anti-CXCR4 antibody, and human immunodeficiency virus infection assays. All 3 methods indicated high affinity of BKT140 for CXCR4, and a one half maximal inhibitory concentration of 1 to $5 \mathrm{nM}$ was determined. ${ }^{19-22}$ Moreover, functional assays have demonstrated that BKT140 treatment blocks CXCL12-induced $\mathrm{Ca}^{2+}$ mobilization and inhibits CXCL12-induced Jurkat $\mathrm{T}$ cell migration in similar one half maximal inhibitory concentrations. ${ }^{19-22}$ Taken together, these findings demonstrate that BKT140 blocks the binding of CXCL12 to extracellular domains of CXCR4 and inhibits CXCR4 signaling. BKT140 also decreases autonomous CXCR4 signaling in CXCR4 wildtype or constitutively active CXCR4 mutants, thus characterizing BKT140 as an inverse CXCR4 agonist. ${ }^{3}$ Similar to other CXCR4/CXCL12 axis-modifying drugs, such as AMD3100 (Mozobil), BKT140 has been shown to be a powerful inducer of hematopoietic stem cell mobilization. ${ }^{18}$ However, a recent report indicated that BKT140, but not AMD3100, showed a CXCR4-dependent cytotoxic effect toward malignant cells of hematopoietic origin. ${ }^{23}$ This suggests that BKT140 could potentially target solid malignancies, but this potential has yet to be defined.

In the present study, we aimed to characterize the in vitro and in vivo therapeutic efficacy of BKT140 against human NSCLC. Using 5 CXCR4-expressing NSCLC cell lines (H358, A549, H460, H1299, and L4), we have demonstrated that BKT140 has both cytostatic and cytotoxic effects on these cells. Furthermore, systemic administration of BKT140 significantly delayed the development of H460-derived tumors and showed a similar trend for A549-derived tumors. Finally, we found that the antiproliferative effects of BKT140 appear to be additive to those of common chemotherapeutic drugs and radiotherapy.

\section{METHODS \\ Cell Lines}

The H460 (large cell carcinoma), A549 (adenocarcinoma), H358 (bronchoalveolar carcinoma), H1299 (large cell carcinoma) cell lines were all purchased from American Type Culture Collection (Manassas, Va) and maintained in Roswell Park Memorial Institute (RPMI) medium (Gibco Laboratories, Grand Island, NY) containing 10\% fetal calf serum (FCS), $1 \mathrm{mM}$ Lglutamine, $100 \mathrm{U} / \mathrm{mL}$ penicillin, and $0.01 \mathrm{mg} / \mathrm{mL}$ streptomycin (Biological Industries, Kibbutz Beth Haemek, Israel). L4 is a primary NSCLC cell line we generated from a large cell carcinoma. ${ }^{15}$ All cell lines were tested for mycoplasma contamination and were negative.

\section{In Vivo Experiments}

We injected $1 \times 10^{6} \mathrm{H} 460$ cells mixed in $100 \mu$ L RPMI 1640 and $100 \mu \mathrm{L}$ matrigel (BD Biosciences, San Diego, Calif) subcutaneously into the right flank of nude mice. Tumor growth was monitored for 19 days. Also, $1 \times 10^{6}$ A549 cells mixed in $100 \mu \mathrm{L}$ RPMI 1640 and $100 \mu \mathrm{L}$ matrigel (BD Biosciences) were injected subcutaneously into the right flank of nude mice. Tumor growth was monitored for 35 days. Systemic BKT140 administration was performed as follows: BKT140 (400 $\mu \mathrm{g} / \mathrm{mouse}$ dissolved in phosphate-buffered saline) was administered once daily 6 or $7 \mathrm{~d} / \mathrm{wk}$ by subcutaneous injection into the left flank at a point at least $1.5 \mathrm{~cm}$ distant from the right-sided tumor. The daily BKT140 injections were initiated on the third day after tumor cell implantation. The tumor size was calculated by measuring the tumor length and width using the following formula: $4 / 3 \times \pi \times($ length $/ 2) \times(\text { width } / 2)^{2}$.

The animal care and use committee at Hadassah University Hospital approved the experimental protocol used in our study.

\section{Giemsa Stain}

A total of $1 \times 10^{5}$ cells $/ \mathrm{mL}$ were cultured in flat-bottom, 24-well plates (Corning, Corning, NY) with $1000 \mu \mathrm{L}$ RPMI 1640 medium supplemented with $10 \% \mathrm{FCS}$ at $37^{\circ} \mathrm{C}$ with $5 \%$ carbon dioxide. Next, 24 hours later, the medium was changed to RPMI 1640 supplemented with $1 \%$ FCS with or without $100 \mu \mathrm{g} / \mathrm{mL}$ BKT140. At the indicated points, the cells were fixed in $100 \%$ methanol for 30 minutes, washed in phosphate-buffered saline, and stained with 1:20 modified Giemsa stain (Sigma-Aldrich, St. Louis, Mo) for 20 minutes. The cells were then rinsed with tap water and images acquired.

\section{Colony Assays}

An agar base layer was prepared as follows: $45 \mathrm{~mL}$ RPMI plus $12 \%$ FCS was mixed with $15 \mathrm{~mL}$ RPMI $\times 2$ plus $12 \%$ FCS and $15 \mathrm{~mL} 2.5 \%$ agar in double-distilled water. The tumor cells were suspended in RPMI plus $10 \%$ FCS. Cell suspension was mixed in a ratio of $1: 3$ with the agar base solution. This mixture was then plated on top of a preformed solid agar base. CXCL12, at concentrations of $25,50,100$, and $250 \mathrm{ng} / \mathrm{mL}$, with or without $100 \mu \mathrm{g} / \mathrm{mL}$ of BKT140, was added to the mixture. Fourteen days later, the number of colonies was counted in 10 different fields.

\section{Immunohistochemistry}

Tissue sections from the tumor-bearing nude mice were routinely fixed with formalin and embedded in paraffin. Antigen retrieval was performed, and the sections were stained with the monoclonal anti-CXCR4 antibody clone 12G5 (R\&D Systems, Minneapolis, Minn) (1:100) using a standard indirect avidin-biotin horseradish peroxidase method according to the manufacturer's instructions. 3-Amino-9-ethylcarbazole was used for color development, and sections were counterstained with hematoxylin. 

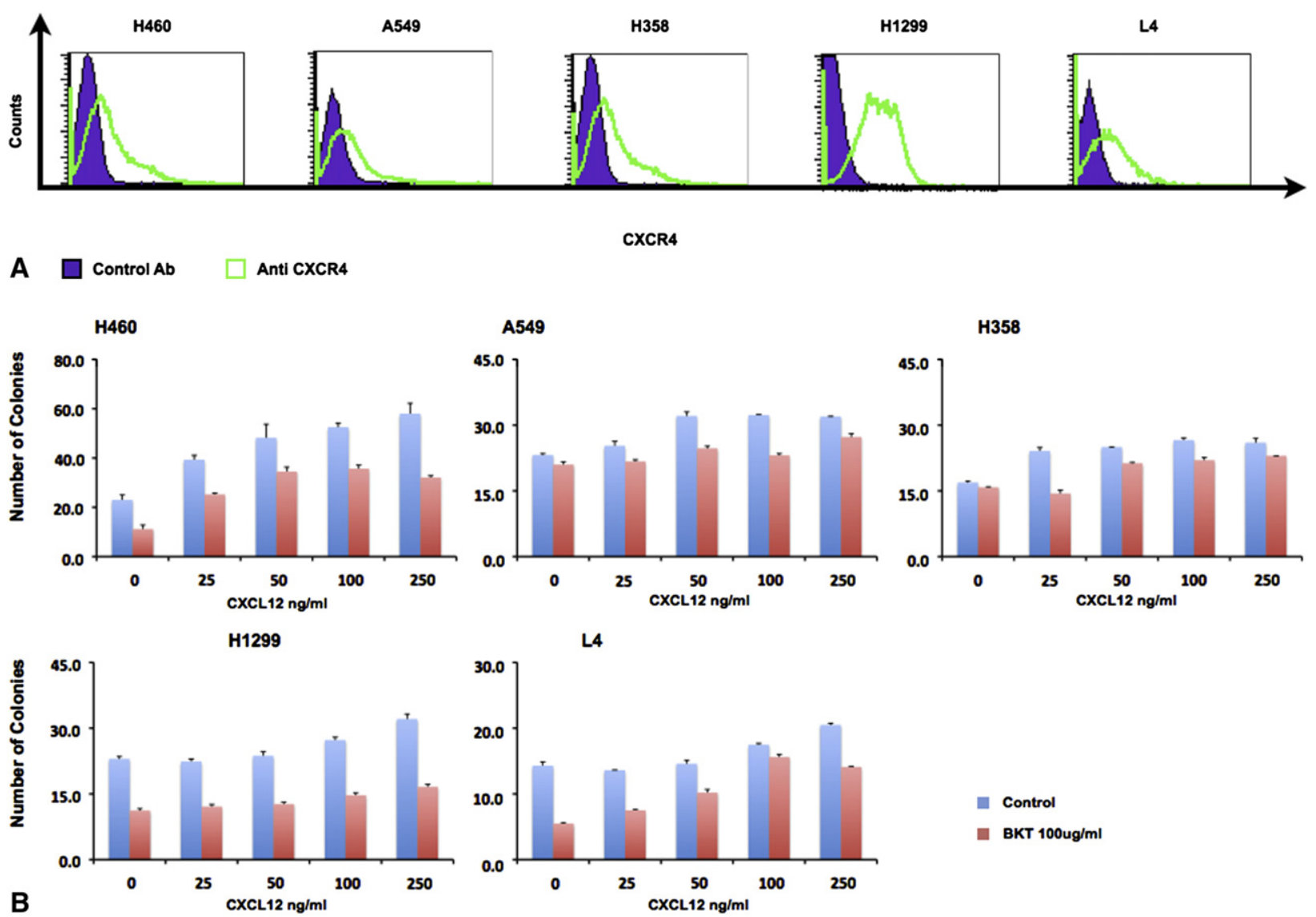

FIGURE 1. A, CXCR4 expression in non-small cell lung cancer cell lines. Representative flow cytometry analyses of CXCR4 staining in H460, A549, H358, H1299, and L4 cells shown. Control antibody (Ab) shown in purple and anti-CXCR4 antibody in green. B, Colony formation by non-small cell lung cancer cell lines in presence of CXCL12 and BKT140. Colony formation by H460, A549, H358, H1299, and L4 cells in response to increasing concentrations of CXCL12 (blue) or increasing concentrations of CXCL12 combined with100 $\mu \mathrm{g} / \mathrm{mL}$ BKT140 (red) shown.

As negative controls, sections were stained with either no primary antibody (phosphate-buffered saline) or an isotype-matched control antibody.

\section{Proliferation Assay}

To measure cell proliferation, 4000 cells were seeded into 96-well cell culture plates with $200 \mu$ L RPMI 1640 medium supplemented with $10 \%$ FCS at $37^{\circ} \mathrm{C}$, with $5 \%$ carbon dioxide. Twenty-four hours later, the medium was changed to RPMI 1640 supplemented with $1 \%$ FCS. Next, 20, 50 , or $100 \mu \mathrm{g} / \mathrm{mL}$ BKT140, with or without cisplatin $0.1 \mu \mathrm{g} / \mathrm{mL}$ or paclitaxel $1 \mathrm{ng} / \mathrm{mL}$ or $0.1 \mathrm{ng} / \mathrm{mL}$ ) was added to the medium. For the radiation experiments, before cell plating, the cells were irradiated at 500 to 2000 cGy. Cultured cells were fixed with $2.5 \%$ glutaraldehyde at the indicated points and stained with $1 \%$ methylene blue. Color extraction was done using $0.1 \mathrm{~N} \mathrm{HCl}$. Absorbance of the solution in each well at $570 \mathrm{~nm}$ was read using a microplate reader (Anthos HTII; Biochrom Anthos, United Kingdom); 6 wells were determined for each measurement point.

\section{Flow Cytometry}

Logarithmically growing cells were harvested and washed, and $1 \times 10^{5}$ cells were stained with antihuman CXCR4 polyclonal anti- $N$-terminus antibody (EMD Millipore, Billerica, Mass) for 30 minutes at $4{ }^{\circ} \mathrm{C}$. PE-labeled donkey antirabbit IgG (eBioscience, San Diego, Calif) was used as a secondary antibody. The incubation time was 30 minutes at $4^{\circ} \mathrm{C}$. The cells were then read and analyzed using a flow cytometry activated cell sorter (Becton Dickinson Immunocytometry Systems; BD Biosciences,
Mountain View, Calif) and CellQuest software. Control staining was obtained by adding $\operatorname{IgG}$ control or only the secondary antibody to the cells.

\section{Survival Assay}

A total of $1 \times 10^{5}$ cells $/ \mathrm{mL}$ were cultured in flat-bottom, 24-well plates (Corning) with $1000 \mu$ L RPMI 1640 medium supplemented with $10 \%$ FCS at $37^{\circ} \mathrm{C}$ and $5 \%$ carbon dioxide. Twenty-four hours later, the medium was changed to RPMI 1640 supplemented with either $1 \%$ FCS, with or without $20 \mu \mathrm{g} / \mathrm{mL}$ or $100 \mu \mathrm{g} / \mathrm{mL}$ BKT140. At the indicated points, the cells were harvested, washed, and stained with propidium iodide solution. The number of live cells was counted and analyzed using a flow cytometry activated cell sorter (Becton Dickinson Immunocytometry Systems) and CellQuest software.

\section{Statistical Analysis}

The data are expressed as the mean \pm standard error and as absolute values. Continuous data with a normal distribution were analyzed using a $t$-test. Categorical data were analyzed using the Mann-Whitney $U$ test. Statistical analyses were performed using SPSS software (IBM, Somers, NY).

\section{RESULTS}

\section{CXCL12 Induced NSCLC Colony Formation in CXCR4-Dependent Manner}

We first characterized the expression of CXCR4 in 5 NSCLC cell lines (H358, A549, H460, H1299, and L4). 
All tested cell lines expressed CXCR4 (Figure 1, A). We next tested the potential of CXCL12 to induce colony formation by these cells. CXCL12 induced a dose-dependent increase in colony formation in all tested cell lines (Figure $1, B$ ). The $\mathrm{H} 460$ cell line was most sensitive to CXCL12 stimulation, with a 2.5 -fold increase in the number of colonies formed in response to $250 \mathrm{ng} / \mathrm{mL}$ CXCL12. The A549 cell line was the least sensitive, with only a 1.4-fold increase in response to similar CXCL12 concentrations. The addition of BKT140 reduced colony formation in all tested cell lines. This observation was evident for the entire range of CXCL12 concentrations. The inhibitory effect of BKT140 on colony formation was most evident in the $\mathrm{H} 460$ and $\mathrm{H} 1299$ cell lines and least evident in the A549 and H358 cell lines.

\section{BKT140 Inhibits NSCLC Proliferation}

The inhibitory effect of BKT140 on colony formation by NSCLC cell lines prompted us to study its potential antiproliferative effects further. Thus, we determined NSCLC proliferation and survival in the presence or absence of increasing concentrations of BKT140. BKT140 treatment inhibited NSCLC proliferation and reduced tumor cell survival in a dose-dependent manner (Figure 2, A). The H460 cell line was most sensitive to BKT140 treatment and the A549 cell line was the least. Treatment with low concentrations of BKT140 $(20 \mu \mathrm{g} / \mathrm{mL})$ was sufficient to completely arrest $\mathrm{H} 460$ tumor cell proliferation, and treatment with high concentrations of BKT140 $(100 \mu \mathrm{g} / \mathrm{mL})$ resulted in tumor cell death. In contrast, treatment with low concentrations of BKT140 $(20 \mu \mathrm{g} / \mathrm{mL})$ did not affect A549 tumor cell proliferation. Nevertheless, treatment with high concentrations of BKT140 $(100 \mu \mathrm{g} / \mathrm{mL})$ significantly reduced A549 tumor cell proliferation and also had some cytotoxic effects. The cytostatic and cytotoxic effects of the low and high concentrations of BKT140 on the H460 and A549 cell lines are also shown in the representative Giemsastained slides presented in Figure 2, $B$.

\section{BKT140 Treatment Delayed NSCLC Tumor Growth In Vivo}

Once we established the in vitro antiproliferative effects of BKT140, we sought to further confirm these observations in vivo. We based our experimental approach on previous publications describing the systemic effects and kinetics of subcutaneous injection of BKT140. ${ }^{24,25}$ As a method to test whether BKT140 injected subcutaneously at a distant site would reach the tumor site, we stained sections of H460 tumors from mice that were untreated or treated with BKT140 for CXCR4. Representative staining of tumor tissue with the control antibody or anti-CXCR4 antibody is shown in Figure 3, $A$ and $B$, respectively. High CXCR4 expression by the tumor cells is evident. Tumor tissue sections prepared from mice treated with BKT140 did not stain for CXCR4 (Figure 3, C). To confirm that this observation had not resulted from transcriptional downregulation of CXCR4 mRNA in the BKT140-treated mice, we performed quantitative real-time polymerase chain reaction for CXCR4 mRNA on both BKT140-treated and -untreated H460-derived tumor tissue samples. We found no effect for BKT140 treatment on CXCR4 mRNA expression levels (Figure E1). Next, we examined the effects of BKT140 treatment on NSCLC tumor development. We focused on the cell lines most sensitive (H460) and least sensitive (A549) to BKT140. BKT140 treatment significantly reduced the volume of H460-derived tumors and showed a similar trend for A549-derived tumors (Figure 3,D and $E$ ). The median tumor volume was decreased by nearly $50 \%$ in both cell lines tested (Figure 3, $D$ and $E)$.

\section{Combining BKT140 With Chemotherapeutic Drugs or Radiation Enhanced Inhibition Efficacy of NSCLC Proliferation}

Current treatment protocols for advanced NSCLC involve concomitant or staged administration of chemotherapeutic agents with radiotherapy. ${ }^{26}$ Among the most widely used chemotherapeutic drugs are cisplatin and paclitaxel. Despite the high initial response rates, the disease often recurs. This has been attributed in part to the increase in chemotherapy and radiation resistance by tumor cells. ${ }^{26}$ Interference with the CXCR4/CXCL12 axis has recently been proposed as a potential strategy to increase tumor sensitivity to chemotherapeutic agents and radiotherapy. ${ }^{7,27}$ Thus, we tested the potential of BKT140 to enhance the cytostatic and cytotoxic effects of cisplatin, paclitaxel, and radiotherapy in the $\mathrm{H} 460$ and A549 cell lines. We first tested the proliferation of H460 and A549 cell lines after escalating radiation doses (Figure $4, A$ ) and in the presence of increasing concentrations of cisplatin (Figure 4, $B$ ) and paclitaxel (data not shown). From these experiments, we defined for each treatment a dose or concentration that showed moderate antiproliferative effects but did not completely inhibit tumor cell proliferation. For radiation pretreatment, the defined dose was $500 \mathrm{cGy}$ (Figure 4, A). For cisplatin, the concentration was $0.1 \mu \mathrm{g} / \mathrm{mL}$ (Figure 4, $B$ ), and for paclitaxel, the concentration was $1 \mathrm{ng} / \mathrm{mL}$ or $0.1 \mathrm{ng} / \mathrm{mL}$ (data not shown). Next, we tested H460 and A549 tumor cell proliferation in the presence of increasing concentrations of BKT140 with or without radiation (Figure 5, A), cisplatin (Figure 5, B) or paclitaxel (data not shown) pretreatment. Overall, an additive antiproliferative effect for BKT140 in conjunction with these treatment protocols was evident (Figure 5, $A$ and $B$ ).

\section{DISCUSSION}

It is well established that CXCL12 induces stimulation of CXCR4, as well as CXCR4 autosignaling enhances tumor 

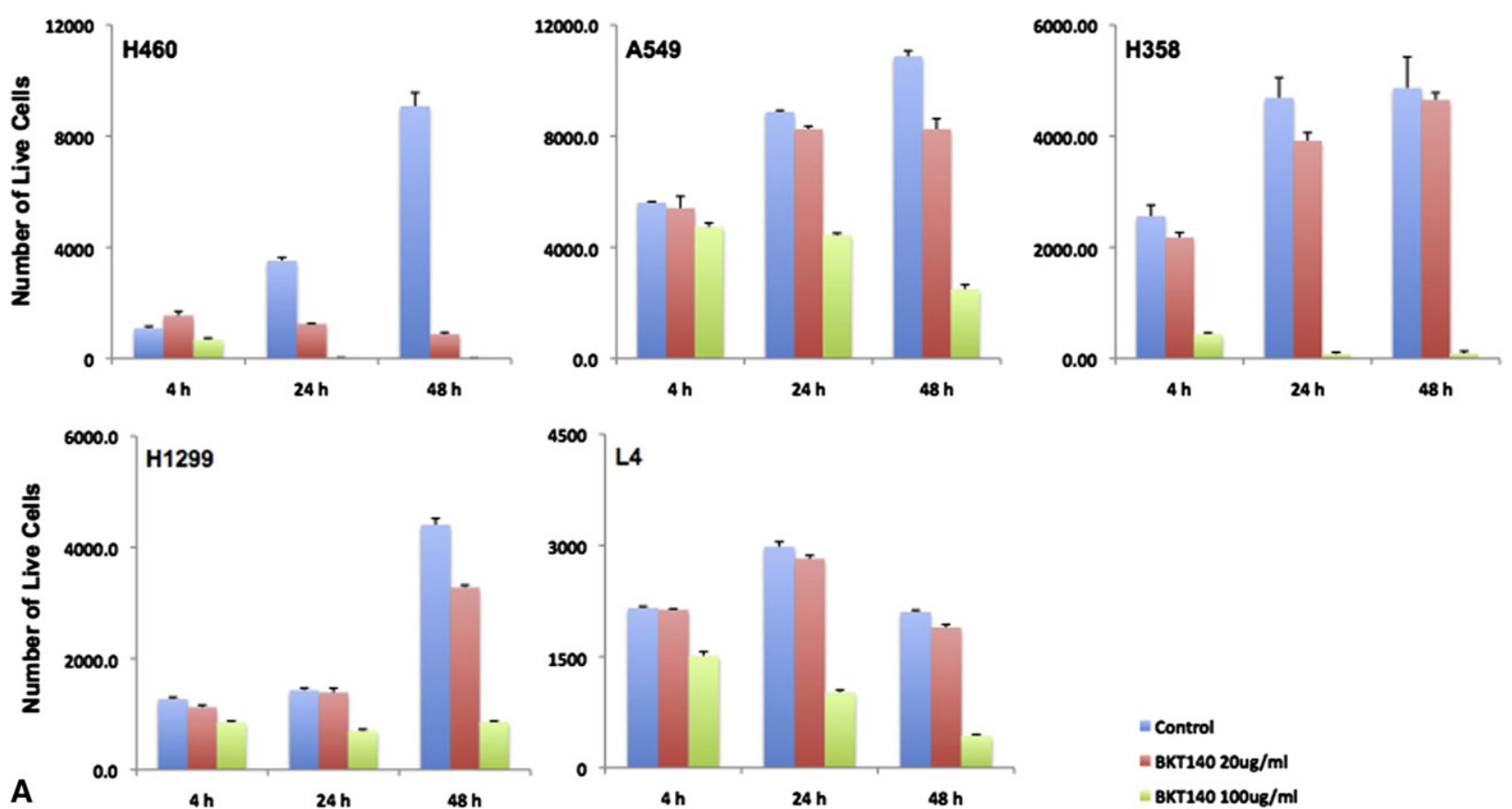

H460

A549
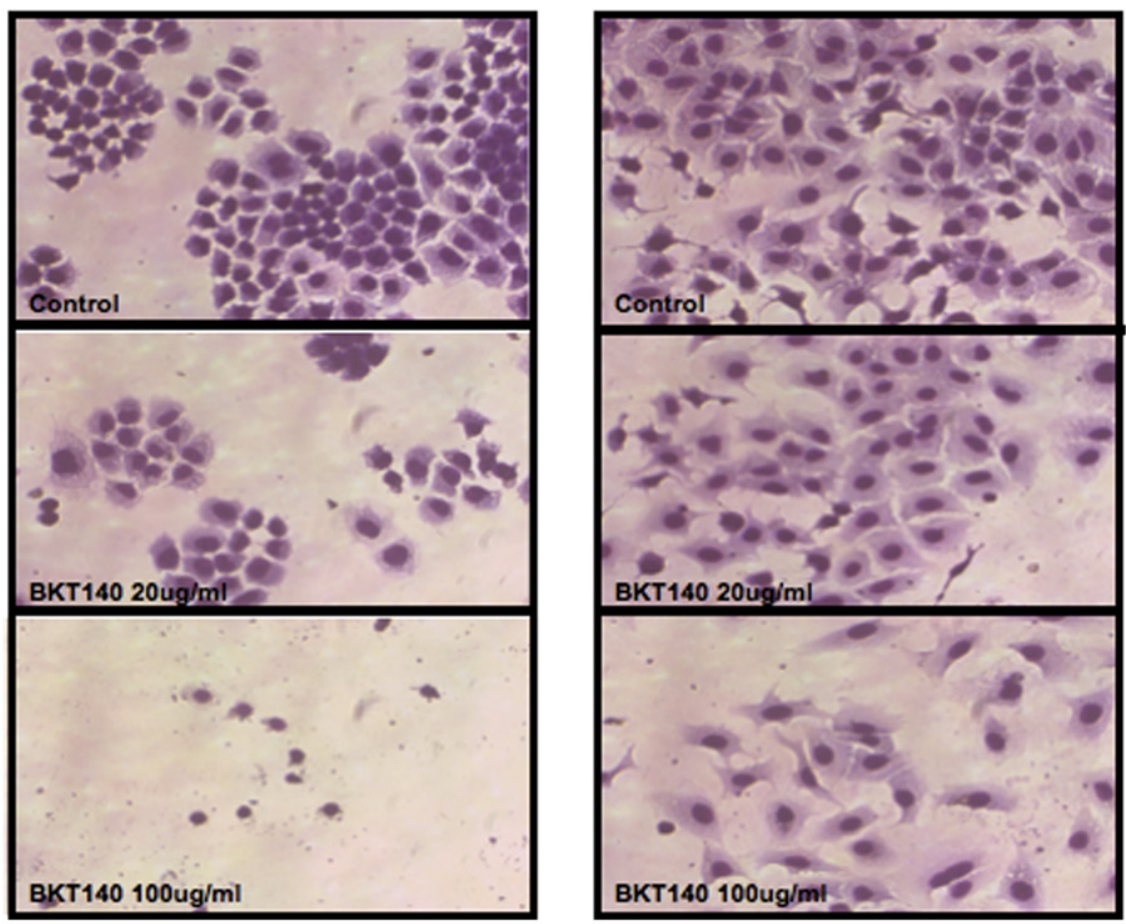

B

FIGURE 2. A, Proliferation of non-small cell lung cancer cell lines in presence of BKT140. Proliferation of H460, A549, H358, H1299, and L4 cells in control medium (blue), medium supplemented with $20 \mu \mathrm{g} / \mathrm{mL}$ (red), or medium supplemented with $100 \mu \mathrm{g} / \mathrm{mL}$ (green) of BKT140 shown. Indicated points were 4, 24, and 48 hours after cell seeding. B, Representative Giemsa staining of H460 and A549 cells growing in control medium or control medium supplemented with $20 \mu \mathrm{g} / \mathrm{mL}$ or $100 \mu \mathrm{g} / \mathrm{mL}$ of BKT140 for 48 hours.

cell proliferation and survival. ${ }^{4}$ Using 5 CXCR4-expressing NSCLC cell lines, we demonstrated that CXCL12 induces NSCLC colony formation in a dose-dependent manner.
Less clear, however, is whether altering or inhibiting CXCR4 signaling would lead to cell cycle arrest or cell death. To address this, we first demonstrated that the addition 

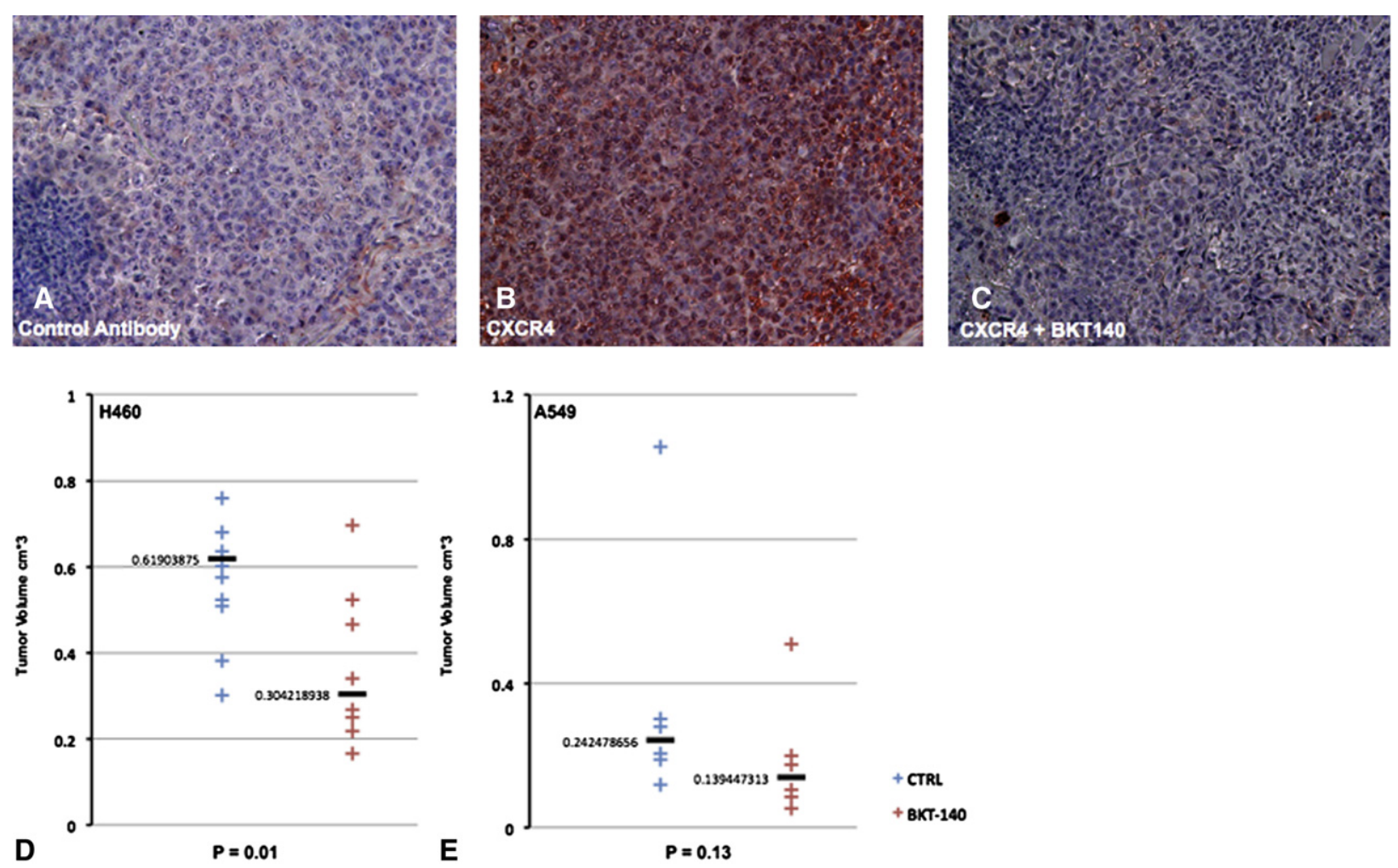

FIGURE 3. Representative staining of H460-derived xenografts for CXCR4. A, Staining with control antibody shown. B, Staining with anti-CXCR4 antibody shown. C, Staining with anti-CXCR4 antibody of xenograft derived from mice receiving daily BKT140 treatment shown. Growth of (D) H460- and (E) A549-derived xenografts with or without daily BKT140 treatment. Tumor volume in mice injected with, Left, H460 cells or, Right, A549 cells shown. Blue crosses indicate control mice (no BKT140 treatment); red crosses indicate mice that received daily BKT140 injections.

of a single dose of BKT140 partially inhibited CXCL12induced colony formation. We also found that the BKT140 reduced the number of colonies forming in the absence of CXCL12 in 3 of the 5 tested cell lines (H460, H1299, and L4). This led us to examine whether BKT140 would delay spontaneous NSCLC tumor cell proliferation in the absence of CXCL12. In line with this prediction, we found that BKT140 had both cytostatic and cytotoxic effects on all tested cell lines. The $\mathrm{H} 460$ cell line was most sensitive to BKT140 and the A549 cell line was the least. Focusing on these cell lines, we demonstrated that a single daily dose of BKT140 was sufficient to induce delayed propagation of H460-derived tumors and, to a lesser extent, of A549derived tumors. Overall, these data have established for the first time the antiproliferative effects of BKT140 against human NSCLC cell lines and tumor xenografts.

Our findings have also demonstrated an enhanced antiproliferative profile for cisplatin and irradiation pretreatment when introduced in conjunction with BKT140. This is of particular importance, because drugs that increase the chemosensitivity and radiosensitivity of malignant cells are continuously being developed to improve the therapeutic outcomes and facilitate reduced dose administration. ${ }^{26}$ The mechanism by which BKT140 adds to the effect of chemotherapeutic drugs and radiotherapy has yet to be elucidated and confirmed in vivo. One intriguing possibility to follow is whether BKT140 specifically targets lung cancer initiating cells (lung cancer stem cells). These cells represent a small subpopulation of tumor cells characterized by high self-renewal capacity, enhanced colony-forming capacity, and superior tumor-forming capacity. ${ }^{28}$ Such cells, which have been identified in both human NSCLC tumors and NSCLC cell lines, are considered unique owing to their resistance to chemotherapeutic drugs and radiotherapy. ${ }^{29-31}$ Preliminary support for the potential role of BKT140 in targeting lung cancer-initiating cells comes from a recent report that has demonstrated that upregulation of CXCR4 is functionally crucial for maintenance of stemness properties in drug-resistant NSCLC cells. ${ }^{16,17}$

Worth mentioning are observations indicating that the vast majority of solid malignant tumors express CXCR4 and that various oncogenic alternations, such as rearranged in transformation/papillary thyroid carcinoma rearrangement, VHL mutation, and TP53 mutation, result in CXCR4 upregulation. ${ }^{4}$ Furthermore, tumor microenvironment-dependent conditions such as hypoxia also specifically induce the upregulation of CXCR4 and CXCL12. ${ }^{4}$ Hence, it is tempting to speculate that the inhibitory effects of BKT140 against NSCLC might also be applicable to other solid malignancies. Our preliminary 


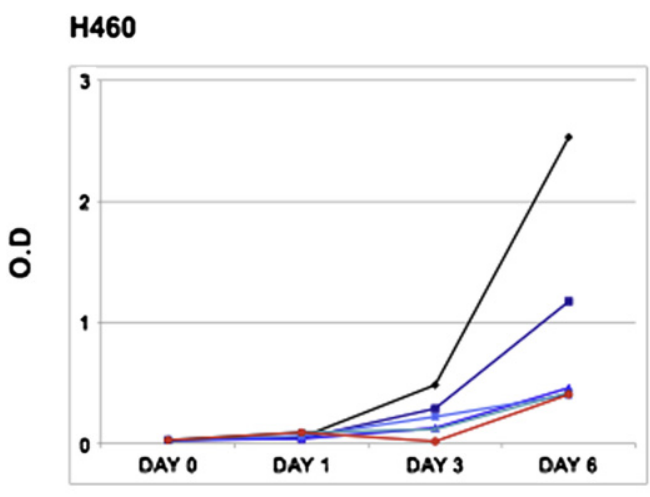

A549
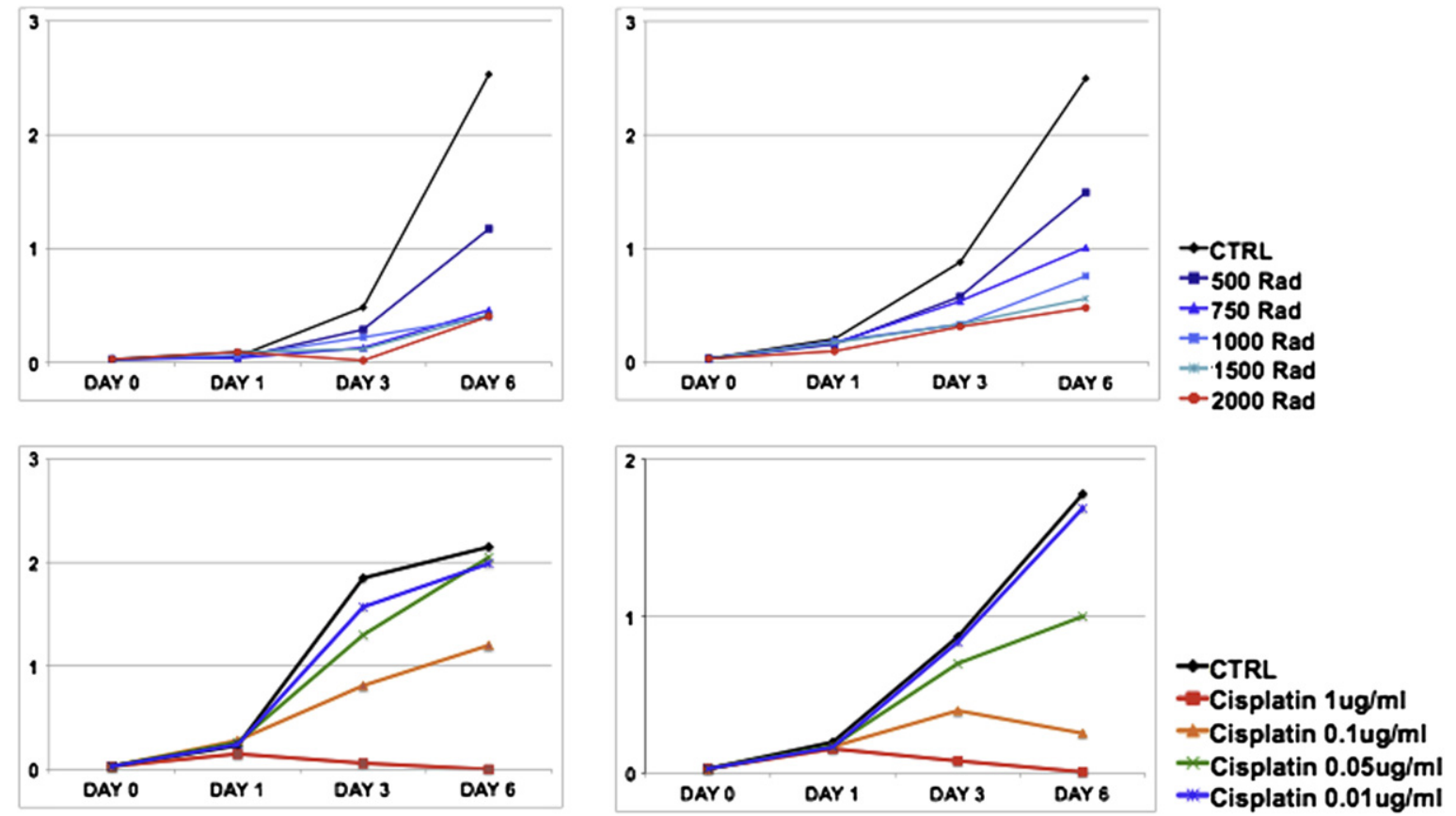

FIGURE 4. Proliferation of $\mathrm{H} 460$ and A549 cells with increasing concentration of cisplatin or increasing radiation doses. Days 0, 1, 3, and 6 shown.

unpublished data have suggested that certain non-NSCLC malignant cell lines are also susceptible to BKT140.

More broadly, CXCR4 is considered a valid target for novel anticancer therapies, and more than 15 new drugs that target the CXCR4/CXCL12 axis are being developed. ${ }^{3,18}$ Several of these drugs, including BKT140, have entered the phase of clinical trial development. ${ }^{3,18}$ Nevertheless, the potential role of CXCR4 antagonists in targeting of NSCLC remains mostly unknown. The sole Food and Drug Administration-approved anti-CXCR4
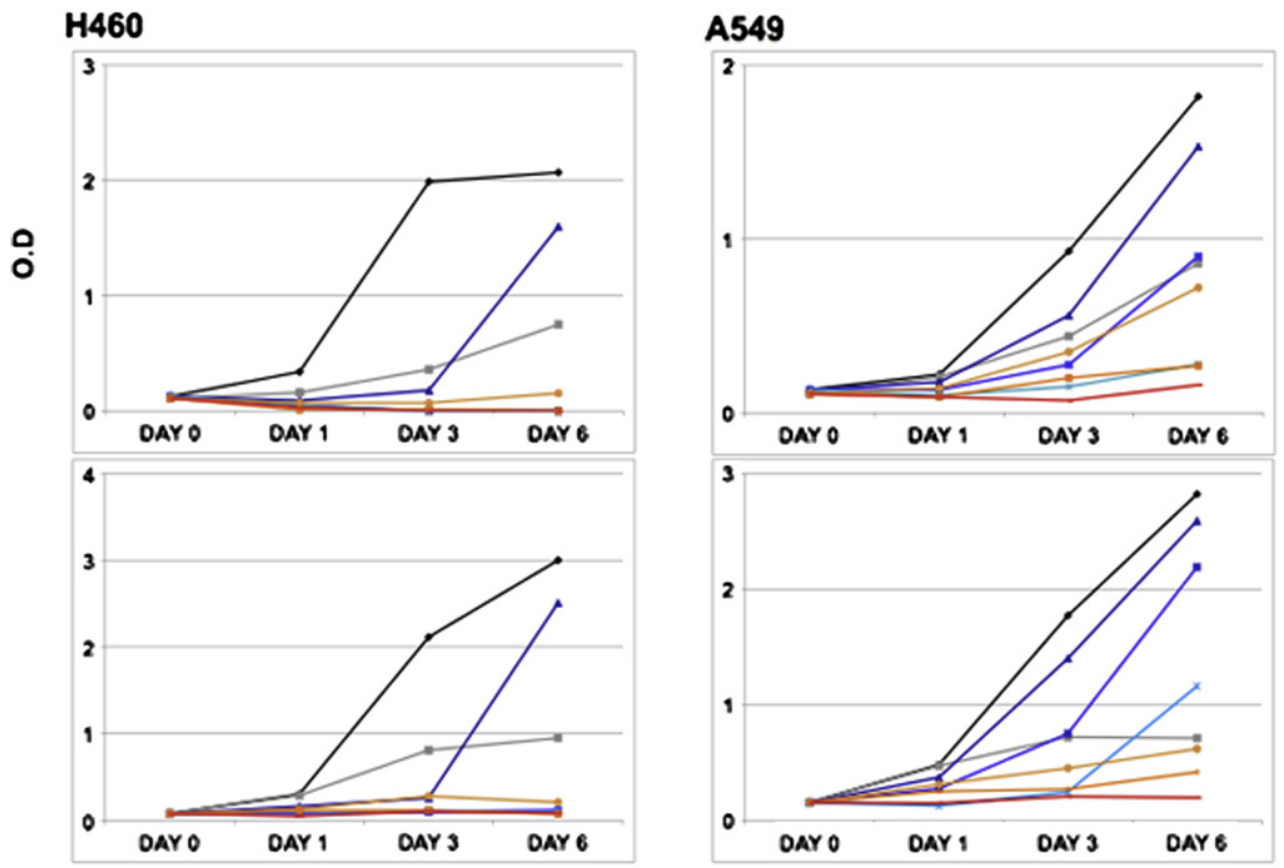

FIGURE 5. Proliferation of $\mathrm{H} 460$ and A549 cells in presence of cisplatin $0.1 \mu \mathrm{g} / \mathrm{mL}$ or after irradiation (500 cGy) with or without increasing concentrations of BKT140. Days 0, 1, 3, and 6 shown. 
agent, AMD3100, was recently shown to suppress the proliferation of a subpopulation of drug-resistant A549 cells. ${ }^{17,32}$ Our work has contributed to the field by demonstrating for the first time the in vitro and in vivo inhibitory effects of BKT140 against a wide range of NSCLC cell lines, representing diverse NSCLC pathologic subtypes, including adenocarcinoma, large cell carcinoma, and bronchioalveolar carcinoma. Squamous cell carcinoma cell lines were not included in the present study, and their susceptibility to BKT140 should be determined in future research. Also, although all tested cell lines expressed CXCR4, they showed a diverse response to BKT140-induced toxicity. Potential explanations for this diverse effect include differential prosurvival/proliferation dependence on CXCR4 signaling among the various cell lines and a lack of absolute specificity of BKT140 for CXCR4, allowing other receptors, such as CXCR7, which is also known to bind CXCL12, to bind BKT140 and modulate its effects. This highlights the necessity in future research to better define BKT140 susceptibility markers. Finally, taking into consideration the importance of the CXCR4/CXCL12 axis in mediating tumor microenvironment interactions, future research will benefit from focusing on the mechanisms by which CXCR4 antagonists such as AMD3100 and BKT140 regulate tumor stroma and tumor immune cell cross-talk.

\section{CONCLUSIONS}

Our findings have indicated that targeting the CXCL12/ CXCR4 axis with BKT140 significantly attenuates NSCLC tumor growth and might augment the effects of antitumor chemotherapy and radiotherapy. These promising results suggest that future research will benefit from delineating the downstream mechanisms of BKT140 action and better defining BKT140 cell susceptibility markers. We believe that by following these paths, scientists and physicians will advance the introduction of CXCR4-based therapeutics for NSCLC, in particular, and solid malignancies, in general.

The authors would like to thank Ishai and Erela Kedem, Rehovot, Israel, and Tomi Nadashi, Haifa, Israel, for their generous support of this research.

\section{References}

1. Hubbard MO, Fu P, Margevicius S, Dowlati A, Linden PA. Five-year survival does not equal cure in non-small cell lung cancer: a Surveillance, Epidemiology, and End Results-based analysis of variables affecting 10- to 18-year survival. J Thorac Cardiovasc Surg. 2012;143:1307-13.

2. Jemal A, Siegel R, Xu J, Ward E. Cancer statistics, 2010. CA Cancer J Clin. 2010;60:277-300.

3. Burger JA, Stewart DJ, Wald O, Peled A. Potential of CXCR4 antagonists for the treatment of metastatic lung cancer. Expert Rev Anticancer Ther. 2011;11: 621-30.

4. Balkwill FR. The chemokine system and cancer. J Pathol. 2012;226:148-57.

5. Zlotnik A, Burkhardt AM, Homey B. Homeostatic chemokine receptors and organ-specific metastasis. Nat Rev Immunol. 2011;11:597-606.
6. Beider K, Abraham M, Begin M, Wald H, Weiss ID, Wald O, et al. Interaction between CXCR4 and CCL20 pathways regulates tumor growth. PLoS One. 2009; 4:e5125.

7. Burger JA, Kipps TJ. CXCR4: a key receptor in the crosstalk between tumor cells and their microenvironment. Blood. 2006;107:1761-7.

8. Mantovani A. Chemokines in neoplastic progression. Semin Cancer Biol. 2004; 14:147-8.

9. Spano JP, Andre F, Morat L, Sabatier L, Besse B, Combadiere C, et al. Chemokine receptor CXCR4 and early-stage non-small cell lung cancer: pattern of expression and correlation with outcome. Ann Oncol. 2004;15:613-7.

10. Su L, Zhang J, Xu H, Wang Y, Chu Y, Liu R, et al. Differential expression of CXCR4 is associated with the metastatic potential of human non-small cell lung cancer cells. Clin Cancer Res. 2005;11:8273-80.

11. Wagner PL, Hyjek E, Vazquez MF, Meherally D, Liu YF, Chadwick PA, et al. CXCL12 and CXCR4 in adenocarcinoma of the lung: association with metastasis and survival. J Thorac Cardiovasc Surg. 2009;137:615-21.

12. Phillips RJ, Burdick MD, Lutz M, Belperio JA, Keane MP, Strieter RM. The stromal derived factor-1/CXCL12-CXC chemokine receptor 4 biological axis in nonsmall cell lung cancer metastases. Am J Respir Crit Care Med. 2003;167:1676-86.

13. Kirshberg S, Izhar U, Amir G, Demma J, Vernea F, Beider K, et al. Involvement of CCR6/CCL20/IL-17 axis in NSCLC disease progression. PLoS One. 2011;6: e24856.

14. Wald O, Izhar U, Amir G, Avniel S, Bar-Shavit Y, Wald $\mathrm{H}$, et al. CD4+CXCR4highCD69+ T cells accumulate in lung adenocarcinoma. J Immunol. 2006;177:6983-90.

15. Wald O, Izhar U, Amir G, Kirshberg S, Shlomai Z, Zamir G, et al. Interaction between neoplastic cells and cancer-associated fibroblasts through the CXCL12/CXCR4 axis: role in non-small cell lung cancer tumor proliferation. J Thorac Cardiovasc Surg. 2011;141:1503-12.

16. Bertolini G, Roz L, Perego P, Tortoreto M, Fontanella E, Gatti L, et al. Highly tumorigenic lung cancer CD133+ cells display stem-like features and are spared by cisplatin treatment. Proc Natl Acad Sci U S A. 2009;106:16281-6.

17. Jung MJ, Rho JK, Kim YM, Jung JE, Jin YB, Ko YG, et al. Upregulation of CXCR4 is functionally crucial for maintenance of stemness in drug-resistant nonsmall cell lung cancer cells. Oncogene, February 27, 2012 [Epub ahead of print].

18. Peled A, Wald O, Burger J. Development of novel CXCR4-based therapeutics. Expert Opin Investig Drugs. 2012;21:341-53.

19. Fujii N, Nakashima H, Tamamura H. The therapeutic potential of CXCR4 antagonists in the treatment of HIV. Expert Opin Investig Drugs. 2003;12:185-95.

20. Tamamura H, Xu Y, Hattori T, Zhang X, Arakaki R, Kanbara K, et al. A lowmolecular-weight inhibitor against the chemokine receptor CXCR4: a strong anti-HIV peptide T140. Biochem Biophys Res Commun. 1998;253:877-82.

21. Jacobson O, Weiss ID, Kiesewetter DO, Farber JM, Chen X. PET of tumor CXCR4 expression with 4-18F-T140. J Nucl Med. 2010;51:1796-804.

22. Jacobson O, Weiss ID, Szajek LP, Niu G, Ma Y, Kiesewetter DO, et al. PET imaging of CXCR4 using copper-64 labeled peptide antagonist. Theranostics. 2011; $1: 251-62$.

23. Beider K, Begin M, Abraham M, Wald H, Weiss ID, Wald O, et al. CXCR4 antagonist 4F-benzoyl-TN14003 inhibits leukemia and multiple myeloma tumor growth. Exp Hematol. 2011;39:282-92.

24. Abraham M, Beider K, Wald H, Weiss ID, Zipori D, Galun E, et al. The CXCR4 antagonist 4F-benzoyl-TN14003 stimulates the recovery of the bone marrow after transplantation. Leukemia. 2009;23:1378-88.

25. Abraham M, Biyder K, Begin M, Wald H, Weiss ID, Galun E, et al. Enhanced unique pattern of hematopoietic cell mobilization induced by the CXCR4 antagonist 4F-benzoyl-TN14003. Stem Cells. 2007;25:2158-66.

26. Chang A. Chemotherapy, chemoresistance and the changing treatment landscape for NSCLC. Lung Cancer. 2011;71:3-10.

27. Burger JA, Peled A. CXCR4 antagonists: targeting the microenvironment in leukemia and other cancers. Leukemia. 2009;23:43-52.

28. Rasheed ZA, Kowalski J, Smith BD, Matsui W. Concise review: emerging concepts in clinical targeting of cancer stem cells. Stem Cells. 2011;29:883-7.

29. Eramo A, Lotti F, Sette G, Pilozzi E, Biffoni M, Di Virgilio A, et al. Identification and expansion of the tumorigenic lung cancer stem cell population. Cell Death Differ. 2008;15:504-14.

30. Rivera C, Rivera S, Loriot Y, Vozenin MC, Deutsch E. Lung cancer stem cell: new insights on experimental models and preclinical data. J Oncol. 2011;2011: 549181.

31. Sutherland KD, Berns A. Cell of origin of lung cancer. Mol Oncol. 2010;4:397-403.

32. DiPersio JF, Uy GL, Yasothan U, Kirkpatrick P. Plerixafor. Nat Rev Drug Discov. 2009;8:105-6. 


\section{Discussion}

Dr Chadrick Denlinger (Charleston, SC). Dr Wald, I enjoyed your presentation. This work represents yet another contribution from you and your laboratory related to the chemokines and chemokine receptors in the development and progression of cancer. The importance of BKT140 and other similar drugs will likely continue to grow as these drugs approach the clinical realm. Your presentation this morning also demonstrates the systemic efficacy of BKT140 in your xenograft studies.

Your presentation focused primarily on the specificity of BKT140 as it affects the CXCR4 receptor. As 1 of your earlier slides demonstrated, there are a number of different chemokine receptors that likely have multiple layers of redundancy related to similar ligand binding but different receptors and overlapping intracellular second messenger systems. In this study, you focused exclusively on the small molecule inhibitors of CXCR4- did you consider also using more specific molecular agents, such as short hairpin loops or siRNAs, as a more specific target for the CXCR4?

Dr Wald. I think that this is an important concern. Actually, siRNA silencing is indeed a great option to demonstrate the specificity. We have had some trouble achieving good transfectants and good silencing of CXCR4 with our cell lines. However, it is known that BKT140 competes with the antibody we used in immunohistochemistry assay for a binding site on the CXCR4 receptor. This is the anti-CXCR4 12G5 clone. Therefore, I believe that we can exclude downregulation of the BKT140. Furthermore, there has been extensive work done with the molecule showing its specificity for CXCR4; nonetheless, we cannot absolutely rule out other possibilities, and, indeed, it is possible that some of the effects of BKT140 might be related to other mechanisms, rather than blocking of CXCR4.

Dr Denlinger. Similarly, all the cell lines used in the study expressed CXCR4. Have you found some NSCLC cell lines that do not express CXCR4 that could be possibly used as negative controls for similar studies?

Dr Wald. The lines we have screened have all had some expression of CXCR4. Furthermore, we observed that when implanted in mice, many cells that do not express CXCR4 tend to upregulate the receptor and express it in vivo.

Dr Denlinger. In previous work from your group, you indicated that it is primarily the fibroblasts that release the CXCL12 that bind to the CXCR4 receptor. In some of the studies presented today, no fibroblasts were involved, yet BKT140 seemed to have antiproliferative effects. How do you explain these results?

Dr Wald. Most of these cell lines used in our experiments also express CXCL12 in vitro, so an autocrine loop is possible.

Another issue that I think is important to consider is that the mechanism by which the drug acts in vitro and in vivo might not be similar. It is reasonable to assume that when cells are transplanted in vivo, CXCR12 and CXCR4 probably regulate multiple processes in the tumor microenvironment. Therefore, I am not certain whether the effect we see in vivo simply represents the inhibitory effect or the cytotoxic effect of the drug ex vivo. Furthermore, I am not certain that all the cytotoxic effects of the drug in vitro are absolutely dependent on blocking the autocrine loop. I believe that this is the case when performing colony assay. In these assays, it has been also shown that other CXCR4 inhibitors, such as AMD300, can block colony formation. However, proliferation is only blocked by BKT140, not by other small molecules that target CXCR4. 


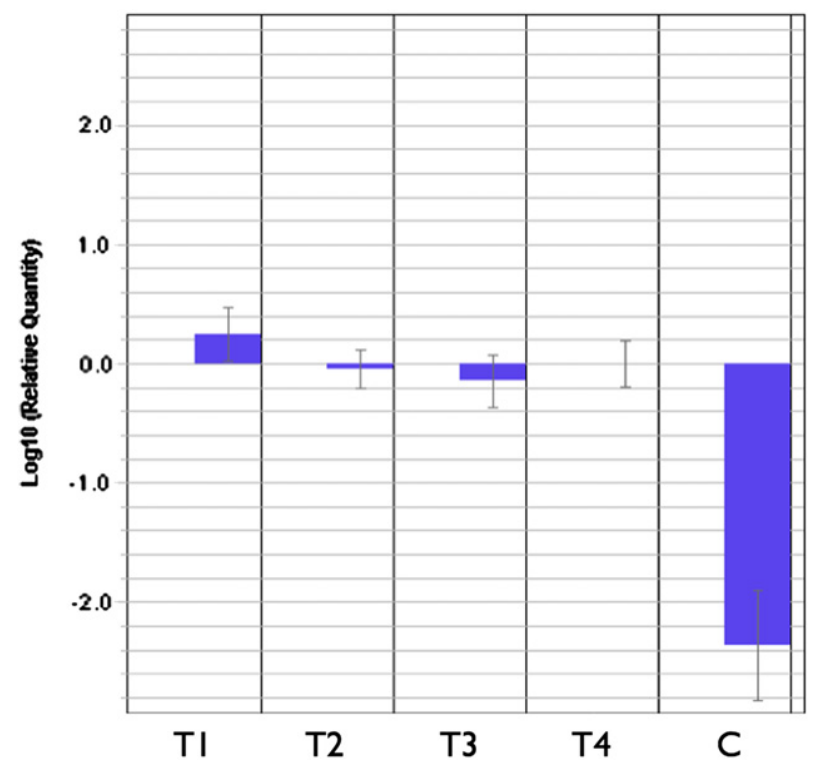

FIGURE E1. Quantitative real-time polymerase chain reaction for CXCR4 mRNA performed on both BKT140-treated and -untreated H460-derived tumor tissue samples and cultured H460 cells. $\log (10)$ of CXCR4 mRNA expression levels in each sample shown. No significant difference were seen in CXCR4 mRNA levels among BKT140-treated and -untreated tumors. T1, H460 tumor after BKT140; T2, H460 tumor after BKT140; T3, H460 tumor control; T4, $\mathrm{H} 460$ tumor control; $C, \mathrm{H} 460$ cell line in vitro. 